\title{
Estudo comparativo com e sem o uso de prototipagem em 3D de uma técnica não convencional no planejamento cirúrgico de revisão de artroplastia total de quadril*
}

Comparative Study with and without the Use of 3D Prototyping of an Unconventional Technique in the Surgical Planning of Revision of Total Hip Arthroplasty

\author{
Alonso Ranzzi ${ }^{1,2}$ Rafael de Luca de Lucena ${ }^{3,4}$ Carlos Roberto Schwartsmann ${ }^{3,5}$ (1)

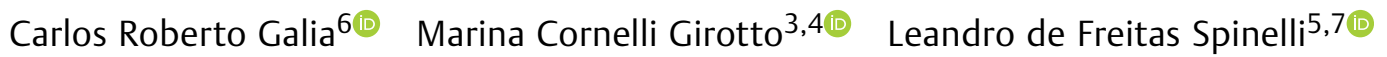

${ }^{1}$ Departamento de Ortopedia e Traumatologia, Santa Casa de Misericórdia de Porto Alegre, Rio Grande do Sul, Brasil

2 Departamento de Ortopedia e Traumatologia, Universidade Federal

de Ciências da Saúde de Porto Alegre, Rio Grande do Sul, Brasil

${ }^{3}$ Serviço de Ortopedia e Traumatologia, Santa Casa de Misericórdia de

Porto Alegre, Rio Grande do Sul, Brasil

${ }^{4}$ Serviço de Ortopedia e Traumatologia, Universidade Federal de Ciências da Saúde de Porto Alegre, Rio Grande do Sul, Brasil

${ }^{5}$ Departamento de Clínica Cirúrgica, Ortopedia e Traumatologia, Universidade Federal de Ciências da Saúde de Porto Alegre, Rio Grande do Sul, Brasil

${ }^{6}$ Departamento de Cirurgia, Ortopedia e Traumatologia, Hospital de Clínicas de Porto Alegre, Universidade Federal do Rio Grande do Sul, Porto Alegre, Rio Grande do Sul, Brasil

7 Programa de Pós-Graduação em Projeto e Processos de Fabricação, Laboratório de Bioengenharia, Biomecânica e Biomateriais,

Universidade de Passo Fundo, Rio Grande do Sul, Brasil
Endereço para correspondência Rafael de Luca de Lucena, MD, Serviço de Ortopedia e Traumatologia, Santa Casa de Misericórdia de Porto Alegre, rua Professor Annes Dias 135, 20 andar - Centro Histórico, Porto Alegre, RS, 90460-150, Brasil (e-mail: rfdldl@gmail.com).

Rev Bras Ortop 2022;57(5):884-890.

Resumo

Palavras-chave

- artroplastia de quadril

- planejamento cirúrgico

- impressão em 3D
Este artigo apresenta uma comparação entre dois casos em que havia a necessidade de revisão de artroplastia total de quadril por falha acetabular asséptica. Utilizamos prototipagem em 3D em um dos casos para realizar uma técnica não convencional de moldagem de material de síntese antes do procedimento, com objetivo de avaliar o tempo economizado no transoperatório em casos complexos.

\footnotetext{
Trabalho realizado no Serviço de Ortopedia e Traumatologia da Santa Casa de Misericórdia de Porto Alegre e na Universidade Federal de Ciências da Saúde de Porto Alegre, Rio Grande do Sul, Brasil.
}

recebido

11 de Outubro de 2020

aceito

11 de Fevereiro de 2021

Publicado on-line

Outubro 13, 2021
DOI https://doi.org/ 10.1055/s-0041-1731659. ISSN 0102-3616.
(C) 2021. Sociedade Brasileira de Ortopedia e Traumatologia. All rights reserved.

This is an open access article published by Thieme under the terms of the Creative Commons Attribution-NonDerivative-NonCommercial-License, permitting copying and reproduction so long as the original work is given appropriate credit. Contents may not be used for commercial purposes, or adapted, remixed, transformed or built upon. (https://creativecommons.org/ licenses/by-nc-nd/4.0/)

Thieme Revinter Publicações Ltda., Rua do Matoso 170, Rio de Janeiro, RJ, CEP 20270-135, Brazil 
Abstract

\section{Keywords}

- arthroplasty, replacement, hip

- surgical planning

- 3D printing
This article presents a comparison between two cases in which there was a need for revision of total hip arthroplasty due to aseptic acetabular failure. We used 3D prototyping in one of the cases to perform an unconventional technique of molding synthesis material before the procedure to evaluate the time saved in the transoperative period in complex cases.

\section{Introdução}

Atualmente, a revisão da artroplastia total de quadril (ATQ) é um procedimento cada vez mais executado. ${ }^{1}$ A complexidade envolvida em cirurgias desse porte frequentemente requer um planejamento pré-operatório meticuloso para que se minimizem os riscos de possíveis complicações associadas.

Embora tenha havido expressiva melhoria dos materiais empregados durante a artroplastia, com o passar dos anos, é inevitável que uma parte considerável dos pacientes tenha a necessidade de trocar os implantes, especialmente devido à soltura asséptica, muitas vezes com falhas acetabulares e femorais consideráveis. ${ }^{2,3}$

Desta demanda aumentada urge a criação e o aperfeiçoamento de técnicas para revisões de ATQ. O planejamento imagiológico pré-operatório progrediu de duas para três dimensões, e, recentemente, surgiram modelagens palpáveis disponíveis em tamanho real ao se utilizar a impressão em 3D. ${ }^{2}$ Isso amplia a compreensão da equipe cirúrgica sobre a patologia em questão, ou seja, expande o entendimento dos envolvidos no procedimento a ser realizado. ${ }^{3}$ Muitos implantes ortopédicos têm um formato padrão que não é compatível com todas situações; então, a moldagem pré-operatória de materiais de síntese pode ser mais adequada à necessidade, além de acelerar $\mathrm{o}$ ato cirúrgico, pois evita esse passo durante a cirurgia, sem que isso de fato aumente consideravelmente os custos finais do procedimento.

Neste sentido, a presente pesquisa realiza um estudo comparativo, e também demonstra uma técnica não convencional no planejamento cirúrgico de revisão de ATQ para avaliar a utilidade prática da prototipagem em 3D para abreviar cirurgias complexas.

\section{Materiais e Métodos}

A presente pesquisa foi submetida ao e aprovada pelo Comitê de Ética em Pesquisa de nossa instituição. $O$ trabalho consta de uma comparação entre dois casos de uma técnica cirúrgica não convencional com e sem o uso de biomodelos, através da utilização de impressão 3D para a revisão de ATQ em que havia necessidade de reconstrução de falha acetabular. Foram selecionados dois casos em que havia sinais radiológicos de soltura asséptica do componente acetabular, e que se encaixavam na classificação 3B de Paprosky.

Os casos operados e relatados neste artigo apresentam uma nova técnica cirúrgica para a reconstrução acetabular. Em um dos casos, utilizamos a prototipagem 3D (PT3D) do quadril que seria operado. A técnica consiste em se obter a imagem 3D da região que será revisada para então ser processada em ambiente de desenho assistido por computador (computer-aided design, CAD, em inglês) e posteriormente impressa. Para tanto, o paciente foi inicialmente submetido a uma tomografia computadorizada (TC) tradicional, que fornece um arquivo computacional no formato de comunicação de imagens digitais em medicina (digital imaging and communications in medicine, DICOM, em inglês), que é então aberto e processado no programa InVesalius (Centro de Tecnologia da Informação Renato Archer, Campinas, SP, Brasil) para conversão ao formato de linguagem padrão de triângulos (standard triangle language, STL, em inglês). Sequencialmente, o arquivo foi editado no programa MeshMixer (Autodesk, Inc., San Rafael, CA, EUA) para a remoção de artefatos, do implante solto, e para melhorar a qualidade da imagem para então ser realizada a impressão do acetábulo em tamanho real com um equipamento 3D Cliever (Cliever Tecnologia, Belo Horizonte, MG, Brasil), utilizando filamento de ácido polilático (polylactic acid, PLA, em inglês). A impressão foi realizada com uma espessura de camada de $0,3 \mathrm{~mm}$, preenchimento de $70 \%$, e espessura de linha de $3 \mathrm{~mm}$, e durou aproximadamente $10 \mathrm{~h}$. A manufatura ocorreu em ambiente controlado com temperatura de $25,8^{\circ} \mathrm{C}$ e umidade do ar de $47 \%$. Após a impressão, um pós-processamento foi realizado para a remoção do excesso de material utilizado no apoio da peça. O custo total de fabricação do biomodelo 3D foi de 78 reais.

O tempo foi cronometrado durante os passos dos planejamentos cirúrgicos pré- e transoperatórios para medida de comparação do objetivo. Estimamos a duração total do tempo da moldagem da placa, o estudo do componente acetabular, o tamanho e a direção aproximados dos parafusos, além da quantidade necessária de enxerto para se preencher a falha acetabular.

Ambas as revisões foram realizadas em apenas um tempo cirúrgico. A via de acesso optada para o segundo procedimento foi a mesma incisão da cirurgia da artroplastia primária, sendo posterolateral nos dois casos.

Uma placa de reconstrução (caixa de pequenos fragmentos) foi moldada em ambos os casos, sendo que, para um deles, utilizamos como base o biomodelo impresso em 3D para realizar os ajustes necessários antes do procedimento, e, para o segundo caso, a modelagem ocorreu no transoperatório. A placa pré-moldada com base no biomodelo em 3D foi esterilizada pelo Setor de Materiais para ser utilizada 
definitivamente durante a cirurgia. Em ambas as reconstruções acetabulares, foram utilizados enxertos ósseos autólogos de ilíaco ipsilateral ao procedimento principal. Ambas as artroplastias foram revisadas utilizando-se um componente acetabular cimentado (polietileno convencional) e cabeça femoral metálica.

Previamente ao procedimento das cirurgias, todos os pacientes foram orientados a respeito da técnica a ser realizada, e concordaram com o processo. Os pacientes foram operados pelos autores do presente artigo.

Os dados dos pacientes foram mantidos em sigilo, servindo apenas para fins de documentação ou ilustração da técnica apresentada.

\section{Descrição do Caso 1}

Uma paciente de 64 anos procurou atendimento por dor crônica progressiva em quadril direito. Havia 10 anos que ela tinha sido submetida à uma ATQ cimentada à direita por fratura do colo do fêmur. No primeiro atendimento, foram realizadas radiografias anteroposterior (AP) de bacia e $\mathrm{AP}+$ de perfil (P) da articulação coxofemoral em questão (- Figura 1A, 1B, 1C). Além disso, solicitamos exames laboratoriais (hemograma, proteína $C$ reativa $[P C R]$, e velocidade de hemossedimentação
[VHS]) que não demonstraram anormalidades. Indicou-se a revisão da artroplastia por soltura asséptica em apenas um tempo cirúrgico (falha acetabular do tipo 3B de Paprosky). A haste femoral não apresentava sinais radiográficos de soltura. $O$ protótipo em 3D (PT3D) foi impresso para o planejamento préoperatório ( - Figura 2) com base em uma tomografia computadorizada da articulação coxofemoral. Utilizando o biomodelo em 3D da pelve, moldamos a placa de reconstrução antes do procedimento, e, então, enviamos para o setor de esterilização para que ela estivesse pronta para ser utilizada no momento da revisão (-Figura 3G). A via de acesso ao quadril direito escolhida foi posterolateral, sendo realizada outra incisão para a retirada de enxerto de ilíaco homolateral (-Figura $\mathbf{3 A}$ ). No transoperatório, testes de estabilidade confirmaram que a haste permanecia fixa, apenas sendo substituída a cabeça metálica femoral. 0 componente acetabular foi extraído, sendo realizado amplo desbridamento local ( - Figura 3B, C, D, E), e posteriormente se posicionou a placa moldada (-Fig. $\mathbf{3 F}$ ), observando-se a mesma cavidade impressa para a modelagem da placa (-Fig. 3G). O tamanho da prótese acetabular também foi estimado durante planejamento utilizando fresas de tamanhos variados (- Figura 4A). Foram utilizados tanto enxerto autólogo estruturado do ilíaco ipsilateral, para evitar
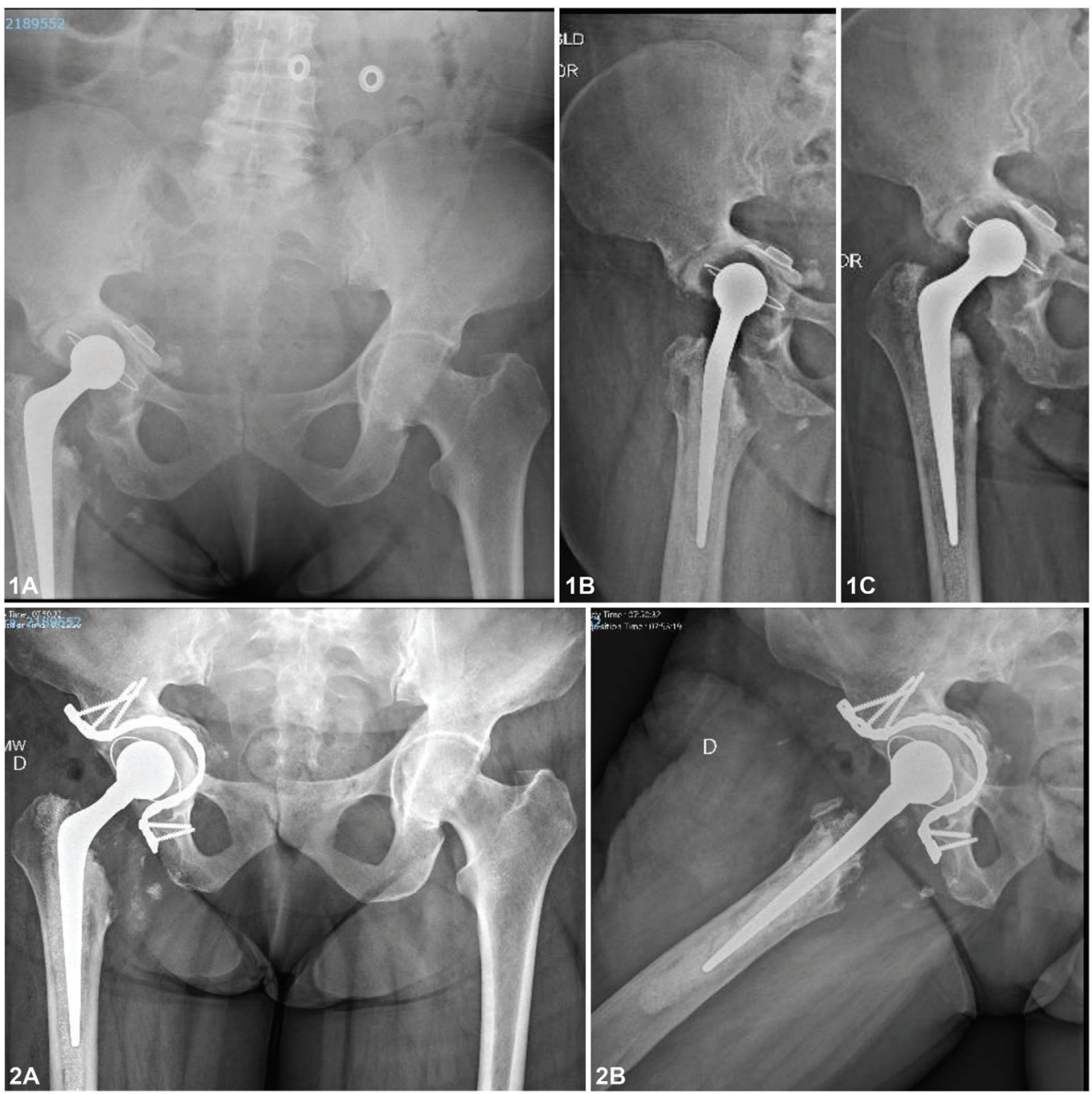

Fig. 1 (A) Radiografias pré-operatórias (caso 1). (B) Radiografias pós-operatórias. 


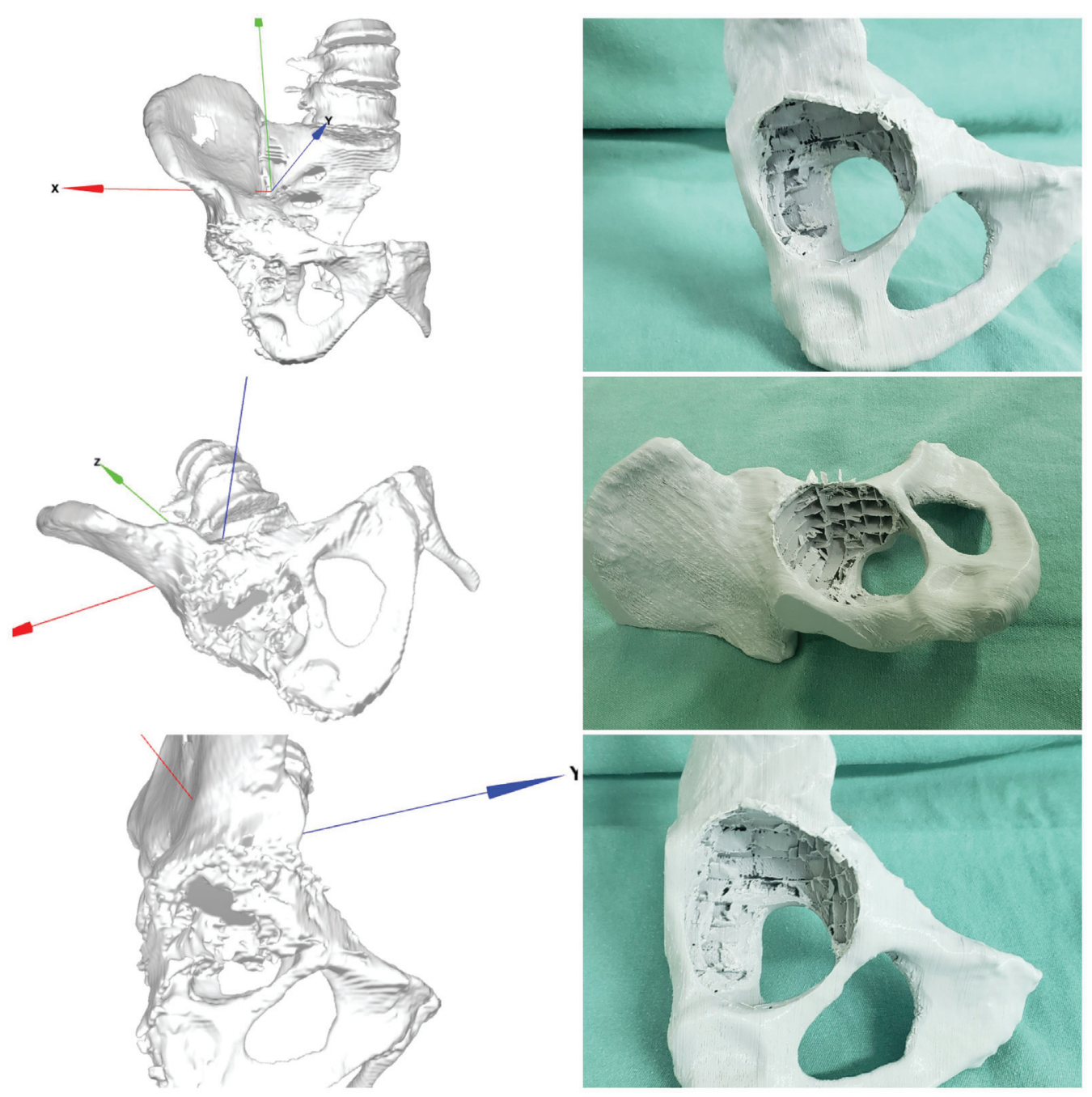

Fig. 2 Prototipagem impressa em 3D (caso 1) após ser processada a partir de tomografia computadorizada.

extravasamento do cimento para dentro da pelve, quanto enxerto esponjoso impactado ao fundo (- Figuras 4B,C). 0 componente acetabular foi cimentado sobre a placa de reconstrução, e o enxerto ósseo e os testes de estabilidade realizados se mostraram adequados (- Figura 4D). O resultado do pósoperatório imediato pode ser visto na -Figura 1B. No momento, a paciente se encontra com 2 anos e 3 meses de seguimento.

\section{Descrição do Caso 2}

Um paciente do sexo masculino, de 58 anos procurou atendimento por dor crônica e limitação funcional em quadril direito. No primeiro atendimento, foram realizadas radiografias $\mathrm{AP}$ da bacia e $\mathrm{AP}+\mathrm{P}$ da articulação coxofemoral em questão (-Figura $\mathbf{5 A}$ ). Indicou-se ATQ por osteoartrose em quadril direito (-Figura 5B). Após 15 anos, ele retornou ao consultório com importante osteólise do acetábulo, classificada como 3B de Paprosky (-Figura 5C), e sinais de osteólise na haste femoral nas zonas de Gruen 1, 2, 6 e 7. Solicitamos exames laboratoriais (hemograma, PCR e VHS) que não demonstraram anormalidades. Optou-se por realizar uma revisão da ATQ em um tempo cirúrgico (- Figura 5D). A haste se mostrou estável durante os testes transoperatórios, e optou-se por mantê-la. Neste momento, o paciente está em acompanhamento há 2 anos (-Figura 5E).

\section{Resultados}

Os casos apresentados demonstram uma nova opção de técnica cirúrgica para resolver os problemas complexos associados à revisão de ATQ para os casos com falhas do tipo 3B de Paprosky. Os pacientes foram operados com sucesso utilizando-se uma placa de reconstrução no fundo acetabular como apoio para enxerto estrutural associado ao impactado para reconstrução do acetábulo para posterior cimentação do componente acetabular da prótese.

O tempo gasto nas moldagens pré- e transoperatória (ou com e sem o uso de impressão 3D, respectivamente) da placa de reconstrução foi, respectivamente, de 8 minutos e 27 minutos até que estivessem adequadamente ajustadas.

A duração da escolha do tamanho do componente acetabular cimentado nos períodos pré- e transoperatória (ou com e sem o uso de impressão $3 \mathrm{D}$, respectivamente) foi de 2 

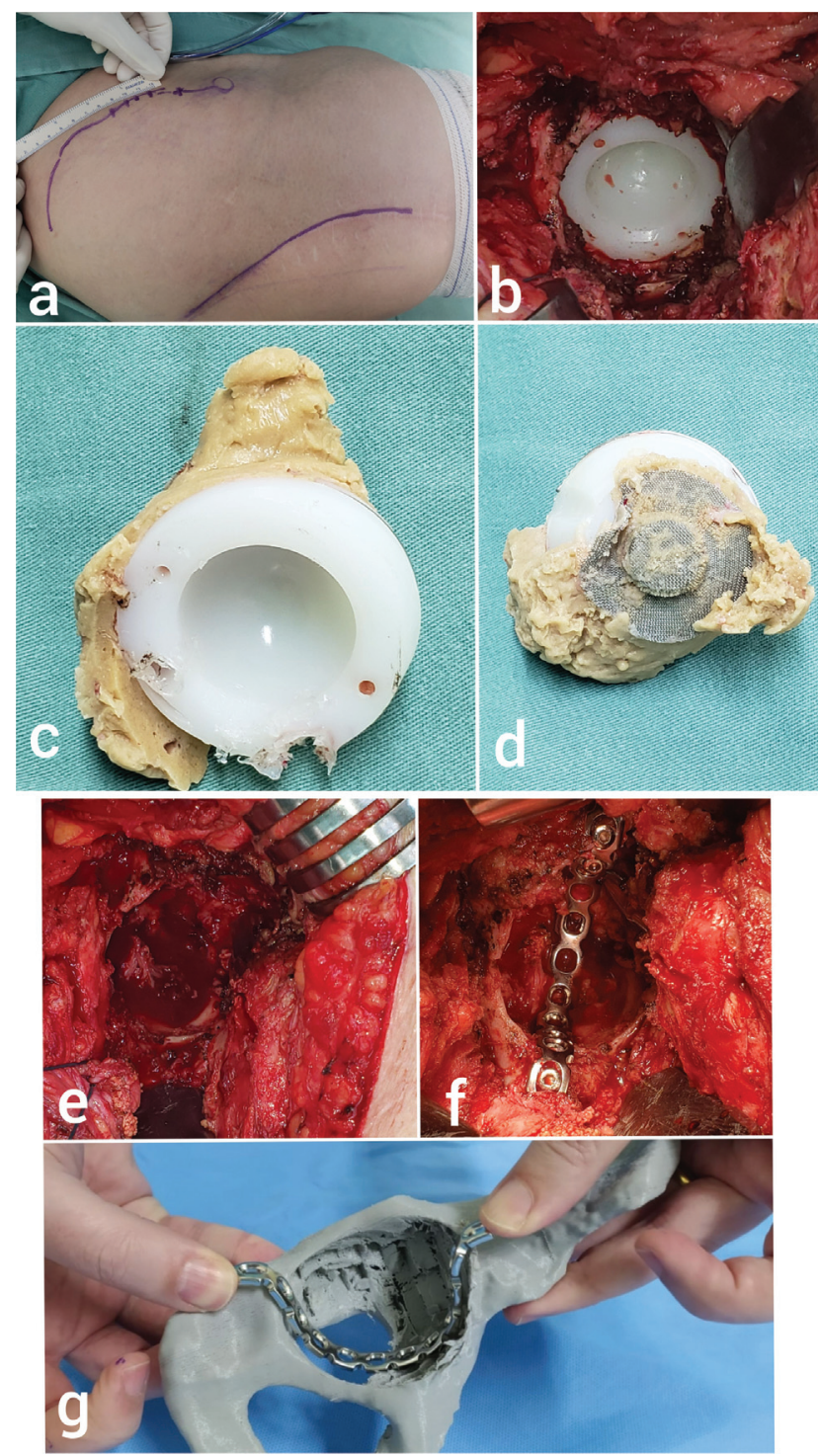

Fig. 3 (A) Incisões operatórias posterolateral e ilíaco ipsilateral (caso 1). (B) Imagem transoperatória do componente acetabular cimentado a ser revisado. (C-D) Componente acetabular retirado. (E) Falha acetabular após a retirada do componente acetabular. (F) Placa de reconstrução moldada encaixada no acetábulo. (G) Planejamento utilizando o biomodelo em 3D com a placa pré-moldada.

minutos e 6 minutos. $O$ tamanho e a direção dos parafusos até a fixação da placa de reconstrução no acetábulo foi, respectivamente de 7 minutos e 25 minutos.

O tempo para se retirar a quantidade de enxerto necessária, sem contabilizar o tempo de extração, foi respectivamente de 3 minutos e 6 minutos (com e sem o uso de impressão 3D). 0 tempo total do procedimento no caso 1 foi de 123 minutos, e, no caso 2, de 179 minutos, uma diferença de 56 minutos.

Durante o transoperatório, não precisamos realizar moldagens adicionais na placa de reconstrução; o componente acetabular cimentado coincidiu exatamente com o planejado segundo os testes na prototipagem, e o tamanho dos parafusos estiveram dentro de uma variação de $\pm 2 \mathrm{~mm}$.

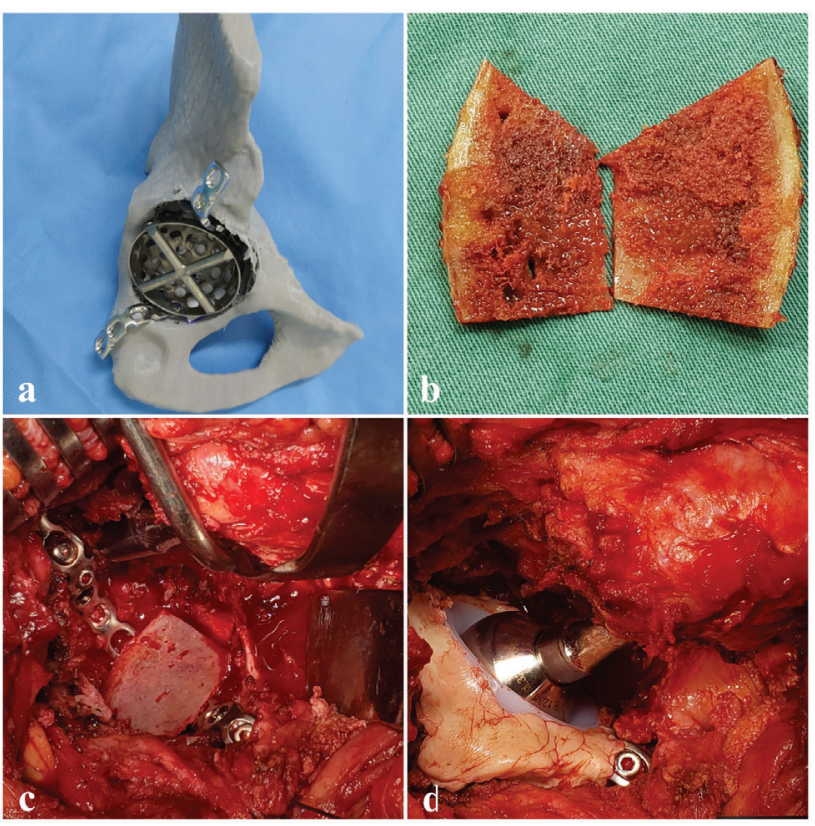

Fig. 4 (A) Placa de reconstrução e fresa acetabular para planejamento pré-operatório. (B) Enxerto ósseo autólogo do ilíaco estruturado em tampa. (C) Tampa de enxerto utilizada no fundo acetabular para evitar o extravasamento de cimento para dentro da pelve. (D) Resultado final do componente acetabular cimentado revisado.

\section{Discussão}

A utilização dos PT3Ds é uma realidade que tem se tornado familiar aos cirurgiões ortopédicos. ${ }^{3}$ Embora não seja uma ferramenta ainda amplamente disponível, a impressão em tamanho real facilita a compreensão e o planejamento do procedimento proposto. Os custos em relação à produção da peça são facilmente suplantados pelo menor tempo cirúrgico, sem levar em conta todos os benefícios sabidos que envolvem um procedimento mais rápido. ${ }^{3-5}$

Outro ponto importante a se ressaltar é que, durante uma revisão de ATQ, frequentemente encontramos uma exposição cirúrgica difícil em função da presença não anatômica dos tecidos fibrosados periprotéticos. ${ }^{5,6} \mathrm{Com}$ a prototipagem, podem-se utilizar marcos anatômicos previamente definidos no biomodelo como referência intraoperatória. Sabemos que nem todas as falhas acetabulares são iguais; portanto, ao realizarmos a moldagem do material, podemos fazer os ajustes necessários. ${ }^{7} \mathrm{~A}$ individualização do tratamento às demandas do paciente é uma tendência médica que reforça a importância do planejamento pré-operatório minucioso, que traz resultados mais confiáveis. ${ }^{2-5}$

A compreensão do paciente é fundamental, sobretudo com relação a uma revisão complexa de ATQ e os desafios encontrados pelo cirurgião, para desmistificar o conceito de que a troca dos componentes é algo muitas vezes simples. Neste sentido, a impressão em 3D pode ser uma ferramenta táctil que auxilia na explicação do procedimento a ser realizado. Embora haja outros pontos positivos a se ressaltar, 

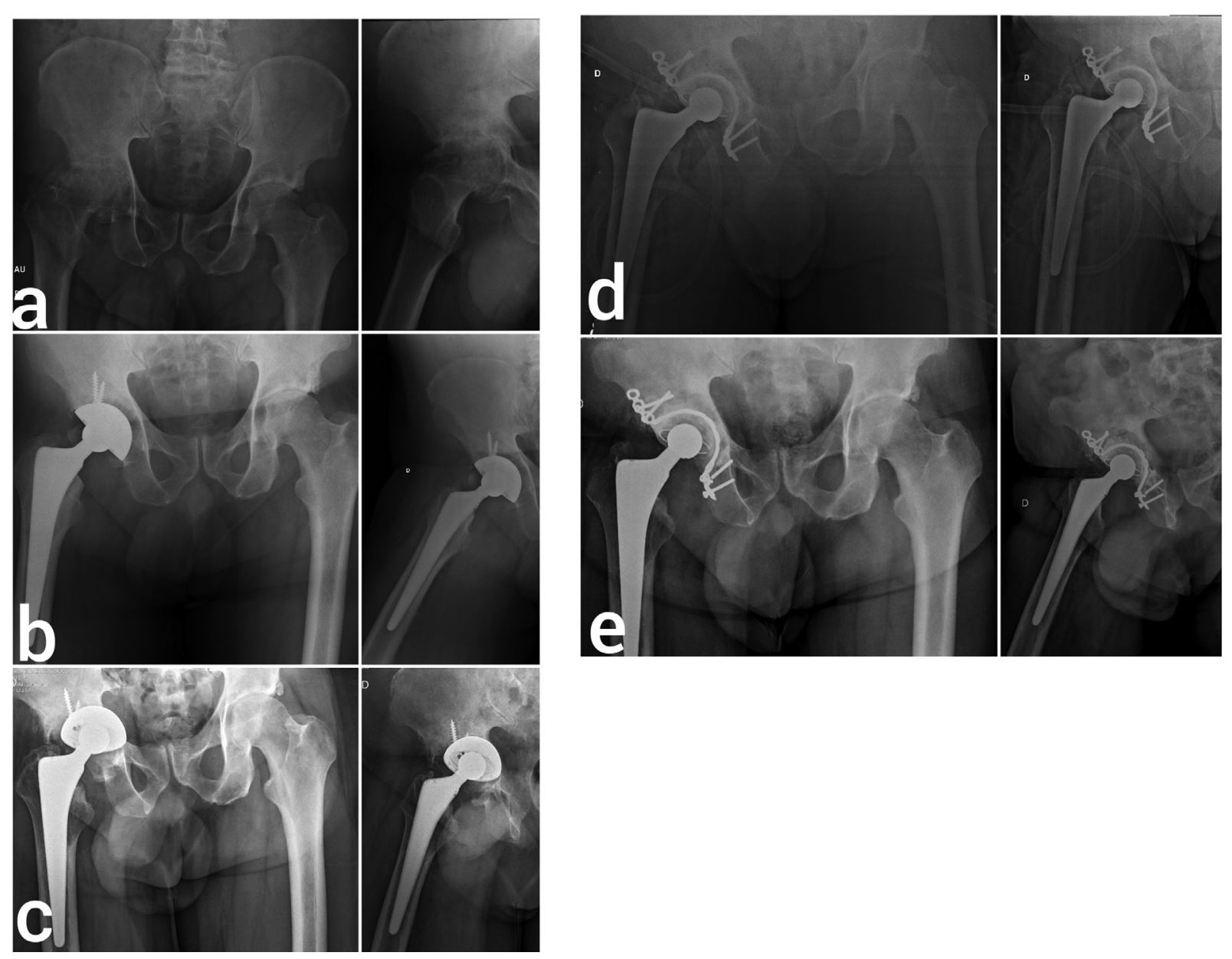

Fig. 5 (A) Radiografia pré-operatória evidenciando importante osteoartrose em quadril direito (caso 2). (B) Radiografia de pós-operatório imediato. (C) Radiografia pós-operatória 15 anos após, com falha acetabular de tipo 3B de Paprosky. (D) Radiografia pós-operatória imediata da revisão. (E) Radiografia pós-operatória com 2 anos de evolução, evidenciando osteointegracão do enxerto no fundo acetabular.

devemos reconhecer, no entanto, que o custo de impressoras 3D ainda é elevado.

Não apresentamos aqui somente uma descrição de dois casos, mas também uma técnica inovadora que tem sido utilizada em nosso serviço, dadas as limitações de materiais que temos no Sistema Único de Saúde (SUS) e a grande demanda por cirurgias de revisão, devido à maior longevidade da população. Por meio dos casos apresentados aqui, demonstramos uma nova opção de técnica cirúrgica para resolver problemas complexos associados à revisão de ATQ para os casos com falhas do tipo 3B de Paprosky. Nestes casos, utilizamos uma placa de reconstrução no fundo acetabular como apoio para enxerto estrutural associado ao impactado para reconstrução do acetábulo. Tal técnica mostrou resultados excelentes até o presente momento. Além disso, o procedimento pode ser otimizado com o uso de impressão em 3D para um melhor entendimento da patologia pela equipe cirúrgica, para o treinamento de residentes, e para a diminuição do tempo cirúrgico e, consequentemente, dos riscos aos pacientes. Entendemos que a casuística ainda é pequena, e que, com os dois casos aqui apresentados, não podemos realizar conclusões genéricas.
Entretanto, a técnica solucionou de forma eficaz os problemas apresentados, e inegavelmente acarretou um menor tempo cirúrgico. Um seguimento mais longo e um número maior de casos ainda são necessários com a técnica proposta, e o uso da prototipagem em 3D pode ser uma alternativa com bom custo-benefício para a resolução de casos específicos.

\section{Comentários Finais}

A técnica apresentada neste artigo para a reconstrução de defeitos do tipo 3B de Paprosky demonstrou excelentes resultados, ainda mais quando associada a alguns dos diversos benefícios da utilização de um PT3D para o planejamento de procedimentos cirúrgicos. A moldagem pré-operatória do material de síntese a ser utilizado pode diminuir o tempo cirúrgico.

\section{Suporte Financeiro}

Não houve suporte financeiro de fontes públicas, comerciais, ou sem fins lucrativos.

\section{Conflito de Interesses}

Os autores declaram não haver conflito de interesses. 
890 Estudo comparativo com e sem o uso de prototipagem em 3D de uma técnica Ranzzi et al.

\section{Agradecimentos}

Os autores agradecem à Dra. Gisele Orlandi Introini, ao Dr. André Peres, a Samuel Werner Wolf, a Luis Fernando Marcelino Braga e a Andreia Gomes Aires do LIPECIN/UFCSPA pela sua importante contribuição para a realização deste trabalho.

\section{Referências}

1 Best JT. Revision total hip and total knee arthroplasty. Orthop Nurs 2005;24(03):174-179, quiz 180-181

2 Xia RZ, Zhai ZJ, Chang YY, Li HW. Clinical Applications of 3Dimensional Printing Technology in Hip Joint. Orthop Surg 2019; 11(04):533-544
3 Fang C, Cai H, Kuong E, et al. Surgical applications of threedimensional printing in the pelvis and acetabulum: from models and tools to implants. Unfallchirurg 2019;122(04):278-285

4 Wyatt MC. Custom 3D-printed acetabular implants in hip surgery-innovative breakthrough or expensive bespoke upgrade? Hip Int 2015;25(04):375-379

5 Woo SH, Sung MJ, Park KS, Yoon TR. Three-dimensional-printing Technology in Hip and Pelvic Surgery: Current Landscape. Hip Pelvis 2020;32(01):1-10

6 Gibon E, Kerboull L, Courpied JP, Hamadouche M. Acetabular reinforcement rings associated with allograft for severe acetabular defects. Int Orthop 2019;43(03):561-571

7 Abdel MP, Trousdale RT, Berry DJ. Pelvic Discontinuity Associated With Total Hip Arthroplasty: Evaluation and Management. J Am Acad Orthop Surg 2017;25(05):330-338 